\title{
Personalized Recommender System using Collaborative Filtering Technique and Pyramid Maintenance Algorithm
}

\author{
Minakshi Pachpatil \\ ME (CSE) \\ Govt.Engg.College ,Aurangabad \\ Maharashtra, India
}

\author{
Anjana N. Ghule \\ Asst.Professer \\ GECA, Aurangabad \\ Maharashtra, India
}

\begin{abstract}
Location aware recommender system(LAS) make use of spatial ratings for generating personalized recommendations.It uses Collaborative filtering techniques to generate recommendations based on user location,item location or both user and item location. LAS uses spatial ratings for spatial items, Spatial ratings for Non-spatial items, Non spatial ratings for spatial items to generate personalized recommendation. For spatial ratings for non spatial items LAS uses user partitioning technique where spatial ratings are distributed as per user location in the pyramid. Pyramid Maintenance algorithm provided to achieve required scalability or locality.LAS is scalable as number of $\gamma$-cells are increased in pyramid and to improve locality $\alpha$-cells are increased to maintain CF Model. LAS is efficient as compare to traditional recommendation system because algorithm provided is strong enough to cope challenge of locality and scalability.
\end{abstract}

\section{Keywords}

Recommender system, spatial location, locality,pyramid structure.

\section{INTRODUCTION}

The goal of recommender system is to generate personalized recommendations for items or products of interest. Suggestions for books on Amazon[1], or movies on Netflix are example of recommender system. Recommender systems make use of Collaborative filtering that make use of past community opinions to find similar users or items to generate number of personalized items. Content based filtering that make use of user profile or description of items. Currently working recommendation system make use of (user, item, rating) attributes which is not produce location based recommendations. Spatial recommender system embeds user litem location with ratings e.g. location based social networks(e.g. Forsquare and Facebook Places[4]) allow check-in at spatial destination \& rate their visits, Sindbad [3] a location based social network system injects location awarenesss within every aspect of social interaction and functionality in the system.LAS produces high quality recommendations using Collaborative filtering in an efficient manner. System produces recommendations using three types of spatial ratings in a single framework. (1)Spatial ratings for non spatial items represented using 4-tuple (user, ulocation, item, rating) where ulocation is the user location, e.g. user at home gives rating to the movie/books/restaurant etc.(2)Nonspatial ratings for spatial items having 4 tuples(user, item, ilocation, rating),here item location is specified.e.g. user from unknown location rating a restaurant/hotels. (3) Spatial ratings for spatial items has 5- tuples(user, ulocation, item, ilocation, ratings).e.g. user at his/her location rating a restaurant visted for lunch.

Two techniques that motivate the need for location aware
Preference locality: preference locality suggests that user ratings from one spatial region are different from ratings in another spatial region. Recommendations should be generated by those ratings which are spatially close to the querying user.

Travel Locality: when recommended items are spatial then user has to travel minimum distance when visiting these venues. This property is termed as "travel locality".

\section{LAS OVERVIEW}

This section provides overview of LAS query model and Collaborative filtering method.

\subsection{LAS Query Model}

Application has given UserId U, K Numeric limit, Location of the user L, then LAS generates K-recommended items for querying user. It can support snap shot queries and continuous queries.

\subsection{Item-based Collaborative Filtering.}

Main idea of collaborative filtering is to use past opinions of user community to predict which item current user will prefer or interested in. Pure collaborative filtering approach takes user-item matrix as a input and produces following types of output. (1) list of n-recommended items which contains those items that user not purchased before. (2) (numerical) prediction indicating to what degree current user will like the items. In Collaborative filtering assumes a set of $\mathrm{n}$ items $\mathrm{I}=\{\mathrm{i} 1, \mathrm{i} 2, \ldots \mathrm{in}\}$ and a set of $\mathrm{m}$ users $\mathrm{U}=\{\mathrm{u} 1, \mathrm{u} 2, \ldots \ldots \mathrm{um}\}$, and each user ui has list of items Iui for which user expressed opinions. Opinions can be numeric rating e.g.(1 to 5 where 1 represents bad choice and 5 represents best choice) or unary rating (e.g.checkboxes) .Active user $\mathrm{Ua}$ for whom the collaborative filtering generates the k-recommended items.

It has two forms.

Prediction: Predicted value is specified in same way as opinions are expressed by active user ua. It is the numerical value expressing predicted likeliness of item ij.

Recommendation: A list of items Ir that active user ua will like most it is the items such that $\operatorname{Ir} \cap$ Iua $=\phi$.

In item based collaborative filtering [9] prediction is computed using similarity between items. Cosine similarity is used to compute similarity as it produces more accurate result. The similarity between two items $\mathrm{a}$ and $\mathrm{b}$ is defined as a rating vector $\bar{a}$ and $\bar{b}$ as shown in fig .2 [8]. Similarity difference is calculated as follows.

$$
\sin (\vec{a}, \vec{b})=\frac{\vec{a} \cdot \vec{b}}{|\vec{a}| *|\vec{b}|}
$$

The possible similarities are between 0 and 1,where 1 indicates strong similarity. After the similarity between the 
items are calculated the next stage is to predict the rating using prediction.For user $\mathrm{u}$ and product $\mathrm{p}$ prediction is calulated as follows.

$$
\operatorname{pred}(u, p)=\frac{\sum_{i \in \text { ratedItems }(u)} \operatorname{sim}(i, p) * r_{u, i}}{\sum_{i \in \text { ratedItems }(a)} \operatorname{sim}(i, p)}
$$

Prediction is the sum of ru,i, a user u's rating for item $\mathrm{i}$ , weighted by $\operatorname{sim}(\mathrm{i}, \mathrm{p})$ similarity of product $\mathrm{p}$ to candidate item $i$,then normalized by similarity score between $i$ and $p$.

User receives as recommendations the top $\mathrm{k}$-item ranked by $\operatorname{pred}(\mathrm{u}, \mathrm{p})$.

\section{SPATIAL USER RATINGS FOR NON- SPATIAL ITEMS}

LAS produces recommendations for spatial ratings for nonspatial items using the tuple(user, uloction, item, rating). Main aim is to achieve preference locality i.e. user opinions are spatially unique, To produce recommendations there are 3 requirements. (1)Scalability: System should be scalable as number of users goes on increasing. (2)Locality: while generating the recommendations consider the ratings of those user spatially close to the querying user. (3)Influence: ability to control the size of spatial neighborhood by system users.User partitioning technique is use to generate recommendations. This technique uses pyramid structure where space is partitioned into different levels of the pyramid as per user location attribute. System then produces recommendations using remaining attributes(user, item, rating). Shape of the pyramid is driven by three goals scalability, influence, locality.

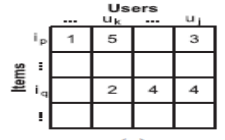

(a)

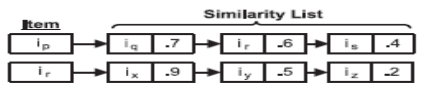

(b)
Fig. 1[8] Item-based CF Model generation. (a) Rating Matrix. (b)Item based CF Model.

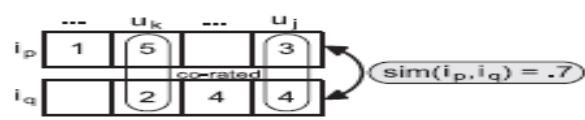

Fig.2. Item-based Similarity calculation.

\subsection{Data Structure}

The pyramid divides the space into $h$ different levels. It make use of partial in-memory pyramid structure[11] as shown in fig.4. For a level h pyramid partitions the space into $4 \mathrm{~h}$ equal area grid cells. At level 0 (root) representing the total geographic area as a one cell. Level 1 partitioned the space into 4 equi-area cells, and so on. Each cell is represented as by unique cell id. As per the need of recommendation locality and scalability pyramid maintains three types of cells. Empty Cell $(\gamma$-cell),Recommendation model cell $(\alpha$-cell $)$, Statistics Cell $(\beta$-cell), Empty Cell $(\gamma$-cell $)[8]$.

Recommendation Model Cell( $\alpha$-cell) Each $(\alpha$-cell) stores an item based collaborative filtering model. This model is built using spatial ratings located in spatial region of that cell. The $\alpha$-cellis the root cell of the pyramid and represent traditional item based collaborative filtering model. $\alpha$-cell maintain items ratings statistics which is in its spatial extents. If $\mathrm{Cp}$ is $\alpha$-cell it contains rating statistics of its 4 cells as shown in fig. 5 . For item i1 ratings located in child cells equal to $109,3200,14,54$.
Statistics Cell( $\beta$-cell) It contains statistics which is within its spatial region. Difference between $\alpha$-cell and $\beta$-cell is that $\beta$ cell does not maintains Collaborative filtering model.It is light weight cell as it require less space as compare to $\alpha$-cell.

Empty Cell $(\gamma$-cell) $\gamma$-cell is a cell that does not maintain statistics and collaborative filtering model. So it is the most light weight cell as it has no CF model. $\alpha$ - cell is responsible for answering recommendation queries as it contains collaborative filtering model. Pyramid structure that contains only $\alpha$-cells achives highest locality. Statistics maintained in $\beta$ - cell determines whether the children of that cell is of $\alpha$ cells for locality point of view. $\gamma$ - cells is leaf cells in the pyramid. Based on the tradeoff between locality and scalability cells are upgrade or downgrade. To achieve locality more $\alpha$-cells are maintained in the pyramid and to achieve scalability more $\gamma$-cells are maintained. $\beta$-cells comes as intermediary cells which further increase the locality where scalability is not affected.

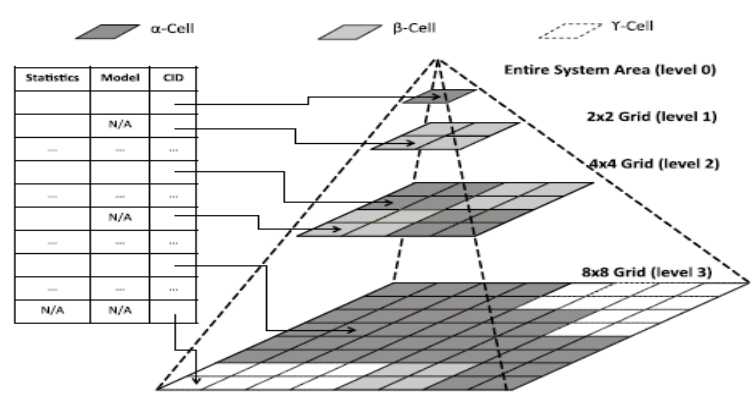

Fig.4 Pyramid Data Structure.

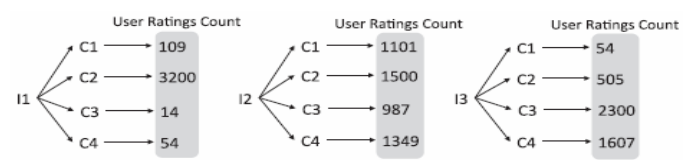

Fig.5[8] Example of Item Ratings Statistics Table.

\subsection{Data Structure Maintainance}

Root cell in the pyramid represents entire region and cells in lowest level representing more localized regions.Initially pyramid is constructed using all spatial ratings and all the cells in the pyramid is $\alpha$-cells. Then invoke cell type maintenance step which scans each cells and downgrades cells to $(\beta-$ cell or $\gamma$-cell) if necessary.

As number of users and items added to the system data will goes on increasing. So that size collaborative filtering model as well as number of recommendations produced from each cell also increasing. Cell maintenance is invoked when cell receives $\mathrm{N} \%$ new ratings, which is computed from the existing ratings.

Features of pyramid maintenance as follows.

(1) Maintenance is performed offline using old pyramid cells. (2) Maintenance does not reconstruct the whole pyramid at once, only one cell is rebuilt at a time.

(3)Maintenance is performed only when $\mathrm{N} \%$ ratings are received by the cell.

\subsection{Maintainance Algorithm}

Algorithm1 provides pseudocode for pyramid maintenance algorithm. It receives input as a Cell $\mathrm{C}$, level $\mathrm{h}$. This algorithm includes three main steps.

Step I: Statistics Maintenance :Parameters in cell C for each item i represents number of user ratings associated with its 
four children.Item ratings statistics table contained ratings which are in spatial extents of cells. If $\mathrm{N} \%$ new ratings received then cell switching decision is made.

Step II: Model Rebuild: As the cell receives new ratings the second step is to rebuild Item based collaborative filtering model. Model is rebuild at cell $\mathrm{C}$ only if cell is $\alpha$-cell. It is necessary to rebuild the model as new ratings enter the system and it should be evovlve in Collaborative filtering model.

Step III: Cell Child Quadrant Maintenance: Pyramid is driven by three goals scalability, influence, locality. Locality and scalability is achieved by cell switching decision. If Cell $\mathrm{C}$ child quadrant cells are $\alpha$-cells then these cells are downgraded to $\beta$-cells by calling function CheckDowngradetoScells. If child quadrant cells are $\beta$-cells then cells are switched to $\alpha$-cells by calling function CheckUpgradetoMCells. If child quadrant cells are $\beta$-cells then LAS first considers to swith the cells to $\alpha$-cells i.e. cell upgradation is done. If cells are not switched to $\alpha$-cells then downgraded to $\gamma$-cells. Cell switching decision is taken completely in quadrants.

\subsubsection{Recommendation Locality}

Running Example : Two level pyramid in which root cell is $\mathrm{cp}$ and it is divided into four cells $\mathrm{c} 1, \mathrm{c} 2, \mathrm{c} 3, \mathrm{c} 4$ shown in fig.6. Eight users $\mathrm{u}=\{\mathrm{u} 1, \mathrm{u} 2, \ldots \ldots, \mathrm{u} 8\}$ and eight items $\mathrm{i}=\{i 1, \mathrm{i} 2, \ldots . \mathrm{i} 8\}$. each user has given ratings to available items. As shown in fig. 6(b) user $\mathrm{u} 2$ and $\mathrm{u} 5$ belongs to cell $\mathrm{c} 2$ both rated the items $\mathrm{i} 2, \mathrm{i5}$. So the similarity score calculated at $\mathrm{c} 2$ is similar to similarity score calculated at parent cell $\mathrm{cp}$. As both the users belongs to same cell. This will not be same if users belong to different cell. LAS loses the locality if CF model produced at child cells different than the CF model at parent cell. System calculates locality gain/lost as follows.

Locality Loss/Gain: Table2 gives mathematical notions used for calculating locality loss/gain. Items ratings pair set (RPc,i) is a set of pairs of users have rated item I in cell c.

e.g. RPcp,i7 is the item rating pair set for item i7 in cell cp with three elements i.e. RPcp,i7 $=\{\langle\mathrm{u} 3, \mathrm{u} 6\rangle,\langle\mathrm{u} 3, \mathrm{u} 7\rangle$ $,<\mathrm{u} 6, \mathrm{u} 7\rangle\}$. For each item define the Skewed Item Rating Set $\mathrm{RSc}$, I which is total number of user pairs in cell $\mathrm{c}$ that rated the item I such that user pairs does not belongs to same cell c. i.e. Skewed item ratings set for i2 in cell $\mathrm{cp}$ is null as users $\mathrm{u} 2$ and $\mathrm{u} 5$ rated item i2 located in same child cell c2. Skewed item ratings set for i4 is $\mathrm{RScp}, \mathrm{i} 4=\{\langle\mathrm{u} 2, \mathrm{u} 7\rangle,\langle\mathrm{u} 7, \mathrm{u} 4\rangle$, $\langle\mathrm{u} 2, \mathrm{u} 4\rangle\}$.Using these parameters calculate the item locality loss LGc,i for each item as follows.

Defination1 Item Locality $\operatorname{Loss}(\mathbf{L G c , i )}$ it is defined as degree of locality loss of item when four children cell of cell c is downgraded to $\beta$-cell, such that $0<=\mathrm{LGc}, \mathrm{i}<=1$.

$$
L G_{C, i}=\frac{\left|R S_{C, i}\right|}{\left|R P_{C, i}\right|} .
$$

Defination 2. Locality Loss(LGc) It is defined as total locality loss of cell $\mathrm{c}$ by downgrading four children cells to $\beta$ cell $(0<=\mathrm{LGc}<=1)$. It is the sum of all item locality loss normalized by total number of items in the cell.

$$
L G_{C}=\frac{\sum_{i \in I_{C}} L G_{C, i}}{\left|I_{C}\right|} .
$$

\section{Summary of Mathematical Notations}

\begin{tabular}{|c|l|}
\hline Parameter & Description \\
\hline \hline$R P_{c, i}$ & The set of user pairs that co-rated item $i$ in cell $c$ \\
\hline$R S_{c, i}$ & $\begin{array}{l}\text { The set of user pairs that co-rated item } i \text { in cell } c \text { such that each } \\
\text { pair of users }\left\langle u_{1}, u_{2}\right\rangle \in S_{c, i} \text { are not located in the same child } \\
\text { cell of } c\end{array}$ \\
\hline$L G_{c, i}$ & $\begin{array}{l}\text { The degree of locality lost for item } i \text { from downgrading the four } \\
\text { children of cell } c \text { to } \beta \text {-Cells, such that } 0 \leq L G_{c, i} \leq 1\end{array}$ \\
\hline$L G_{c}$ & $\begin{array}{l}\text { The amount of locality lost by downgrading cell } c \text { four children } \\
\text { cells to } \beta \text {-Cells }\left(0 \leq L G_{c} \leq 1\right)\end{array}$ \\
\hline
\end{tabular}

cell locality loss determines whether cell children need to downgrade from $\beta$-cell to $\gamma$-cell, or upgrade from $\gamma$-cell to $\beta$ cell, downgraded from $\alpha$-cells to $\beta$-cell. Which are explained as follows.

\section{Downgrading $\alpha$-cells to $\beta$-cell}

This operation downgrades the entire cell quadrant at level $h$ from $\alpha$-cells to $\beta$-cell having common parent at level h- 1 . Downgrading the cells to $\beta$-cell improves the scalability with two performance improvement (1)Continuous query processing computation is less as it does not maintain $\mathrm{CF}$ model and no need to update recommendation query answer as it crosses boundry of $\beta$-cell if it covers large spatial region. (2) low maintenance cost as $\beta$ - cells does not contain $\mathrm{CF}$ model so less CF models are built periodically.To downgrade the cells to $\beta$-cells call the function CheckDowngradeToSCells in Algorithm1. It require two percentage values (1)Locality Loss as described in prevous section.(2) Scalability gain,the amount of scalability gain by downgrading the cells. LAS Downgrades the cells to $\beta$-cell if

\section{$(1-M) *$ Scalability_gain $>M *$ locality_loss}

$M$ is a real number having range[0,1]. Large value of $M$ is use to achieve locality, Scalability is achieved by small value of M.

Scalability Gain: Scalability gain is calculated by the summation of recommendation model size of cells to be downgraded(sizem) divides this value by sum of sizem and model size of parent cell.

Cost: Using Item Ratings statistics table at cell cp the locality loss is calculated inO( $|\mathrm{Icp}|)$ time, where Icp is the total number of items in the cell. Time required to calculate scalability gain is $\mathrm{O}(1)$. i.e. total time cost required to downgrade cells to $\beta$-cells is $\mathrm{O}(|\mathrm{Icp}|)$.

\section{2) Upgrading $\beta$-cells to $\alpha$-cells}

Upgrading $\beta$-cells to $\alpha$-cells increase the recommendation locality. It requires to maintain $\mathrm{CF}$ model at each children cells which need to be upgrade. It hurts the scalability because more maintenance and storage is required for CF model at each child cell. To determine whether the child cells are need to be upgrade two perecentage values are calculated that is locality gain and scalability loss. Child cells of cell $\mathrm{cp}$ are upgrade if following condition holds.

\section{$M *$ locality gain $>(1-M) *$ Scalability loss}

It is opposite to downgrading $\alpha$-cells to $\beta$-cell. Locality gain is calculated same way as the locality loss is calculated. Scalability loss is estimation of storage required to maintain CF model at child cells. For item based CF Model the maximum size is $n[\mathrm{I}]$. $\mathrm{n}$ is model size. By multiplying $\mathrm{n}[\mathrm{I}]$ to the number of bytes required to store an item to find upper bound of storage size. Scalability loss is calculated by the sum 
of These four estimated sizes (sizes) divided by the sum of existing parent cell and sizes.Time required to calculate scalability loss is $\mathrm{O}(1)$ and time required to calculate locality gain is $\mathrm{O}(|\mathrm{Icp}|)$. Total time cost to upgrade cells to $\alpha$-cell is $\mathrm{O}(|\mathrm{Icp}|)$.

\section{3) Upgrading $\beta$-cells to $\alpha$-cells}

Upgrading $\beta$-cells to $\alpha$-cells increase the recommendation locality. It requires to maintain $\mathrm{CF}$ model at each children cells which need to be upgrade. It hurts the scalability because more maintenance and storage is required for $\mathrm{CF}$ model at each child cell. To determine whether the child cells are need to be upgrade two perecentage values are calculated that is locality gain and scalability loss. Child cells of cell $\mathrm{cp}$ are upgrade if following condition holds.

\section{$M *$ locality gain $>(1-M) *$ Scalability loss}

It is opposite to downgrading $\alpha$-cells to $\beta$-cell. Locality gain is calculated same way as the locality loss is calculated. Scalability loss is estimation of storage required to maintain CF model at child cells. For item based CF Model the maximum size is $n[I] . n$ is model size. By multiplying $n[I]$ to the number of bytes required to store an item to find upper bound of storage size. Scalability loss is calculated by the sum of These four estimated sizes (sizes) divided by the sum of existing parent cell and sizes.Time required to calculate scalability loss is $\mathrm{O}(1)$ and time required to calculate locality gain is $\mathrm{O}(|\mathrm{Icp}|)$. Total time cost to upgrade cells to $\alpha$-cell is $\mathrm{O}(|\mathrm{I} c \mathrm{p}|)$.

\section{4) Downgrades $\beta$-cells to $\gamma$-cells and vice versa}

In this $\beta$-cells of children quadrant $\mathrm{q}$ at level $\mathrm{h}$ is downgraded to $\gamma$-cells. It has same parent at upper level.It does not

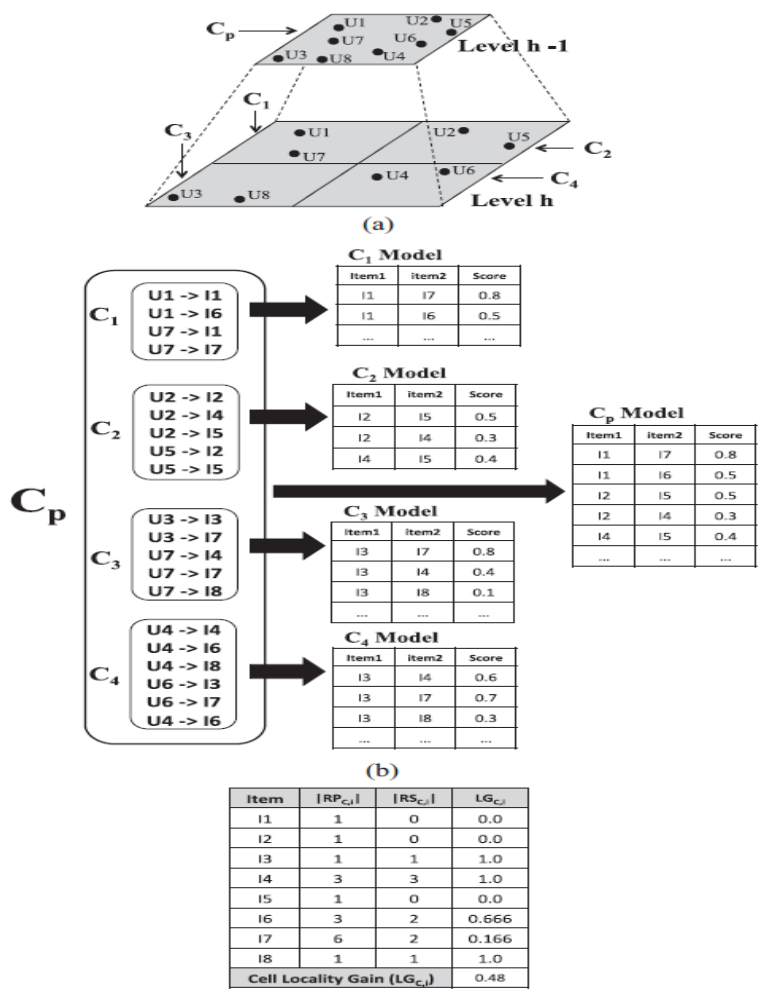

(c)

Fig 6[8].(a) Two Level Pyramid. (c) Locality loss/gain. (b) Recommendation model and Item ratings distribution. The value of $R S c, I$ and $R P c, I$ is derived from item ratings statistics table.Value of $\mathbf{L G c , i}$ is used to calculate overall cell locality loss. required overhead of storing item ratings statistics. It reduce the amount of locality. Decision is taken based on MAX_SLEVEL, maximum number of consecutive pyramid levels. The value is between 0 and total height of the pyramid. A high value of MAX SLEVEL causes maintaining more $\beta$ cell and less $\gamma$-cell. As shown in fig. 4 two levels of pyramid are $\beta$-cell and third level will automatically set to $\gamma$-cell. For each $\beta$-cell there is $S$-Level counter which stores the consecutive number of $\beta$-cell levels. If its children cells are $\beta$ cell then MAX_SLEVEL is compared with S_LEVEL counter, at a $\beta$-cell. The counter counts only when consecutive level contains $\beta$-cell if it encounter $\alpha$-cells counter reset to zero. If S_LEVEL counter is greter than or equal to MAX_SLEVEL then children cells downgraded to $\gamma$ cells.Similarly S_LEVEL counter is use to upgrade $\gamma$-cells to $\beta$-cell.

\section{EXPERIMENTS}

This section provides experimental evaluation of system based on an actual system implementation using java.Experiments are based on following dataset:

\subsection{Dataset}

Dataset name MH-Hotels is created which consist of list of hotels in Maharashtra, India. Users list include userid, user name, location.Venues list include hotelid, hotel name, location. Rating list contains userid, venueid and ratings( 1 to 5)where 1 is lowest rating and 5 is highest rating. Pyramid is constructed so that it will generate more localized recommendations by maintaining more $\alpha$-cells using algorithm1.cells are downgrade or upgrade as per the requirement of localization using pyramid maintenanace algorithm.

We divided the database into a training set and a test set. For this purpose, we introduced a variable that determines what percentage of data is used as training and test sets; we call this variable $x$. A value of $x=0: 8$ would indicate $80 \%$ of the data was used as training set and $20 \%$ of the data was used as test set.

\subsection{Evaluation Metrics}

Recommender systems research has used several types of

measures for evaluating the quality of a recommender system. They can be mainly categorized into two classes:

Statistical accuracy metrics evaluate the accuracy of a system by comparing the numerical recommendation scores against the actual user ratings for the user-item pairs in the test dataset. Mean Absolute Error (MAE) between ratings and predictions is a widely used metric. MAE is a measure of the deviation of recommendations from their true user-specied values. For each ratings-prediction pair < pi; qi > this metric treats the absolute error between them, i.e., |pi,qi| equally. The MAE is computed by _rst summing these absolute errors of the $\mathrm{N}$ corresponding ratings-prediction pairs and then computing the average. Formally,

$$
M A L=\frac{\sum_{i=1}^{N}\left|H_{i}-t i b\right|}{N}
$$

The lower the MAE, the more accurately the recommendation engine predicts user ratings.

Decision support accuracy metrics evaluate how effective a prediction engine is at helping a user select high quality items from the set of all items. These metrics assume the prediction process as a binary operation either items are predicted (good) 
or not (bad). With this observation, whether a item has a prediction score of 1:5 or 2:5 on a five-point scale is irrelevant if the user only chooses to consider predictions of 4 or higher.

We used MAE as our choice of evaluation metric to report prediction experiments because it is most commonly used and easiest to interpret directly.

\subsection{Experimental Results}

In this section we present our experimental results of applying item-based collaborative fltering techniques for generating predictions. Our results are mainly divided into two parts quality results and performance results.

\subsubsection{Quality Experiments}

\section{Recommendation Quality for Varying Pyramid Levels:}

These experiments test the recommendation quality improvement that LAS achieves over the standard (nonspatial) item-based collaborative filtering method.

Quality Metric To measure quality, we build each recommendation method using $80 \%$ of the ratings from each

data set. For each rating $\mathrm{t}$ in this $20 \%$, we request a set of $\mathrm{k}$ ranked recommendations $S$ by submitting the user and ulocation associated with $\mathrm{t}$. We first calculate the quality as the weighted sum of the number of occurrences of the item associated with $t$ (the higher the better) in S. The weight of an item is a value between zero and one that determines how close the rank of this item from its real rank.

Fig. compares the quality improvement for varying locality (i.e., different levels of the adaptive pyramid) using the our dataset. The quality of LARS increases as more localized pyramid cells are used to produce recommendation, which verifies that user partitioning is indeed beneficial and necessary for location-based ratings.

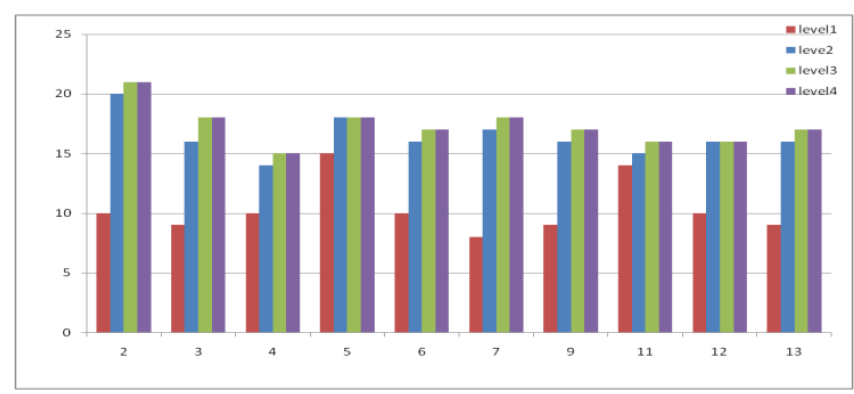

Fig.7 Quality Experiments for varying locality.

\section{Recommendation Quality for Varying $M$}

These experiments compares the quality improvement achieved by both LARS and LAS for different values of $M$. Fig. depicts the effect of M on the quality of LARS using our data set. We report quality numbers using the pyramid height of four.

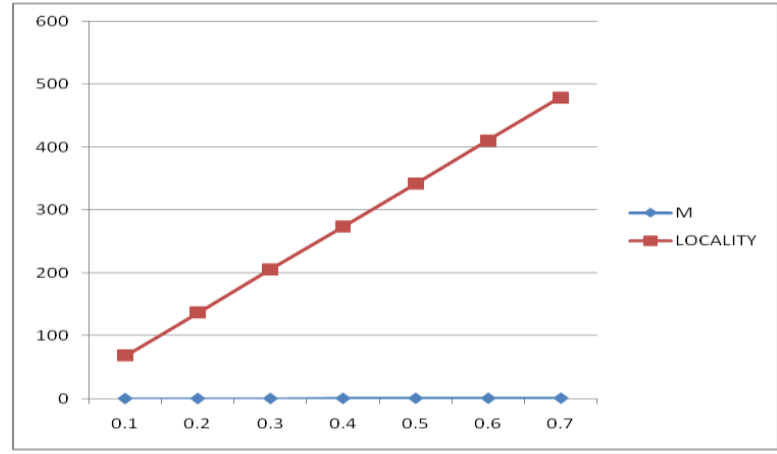

Fig.8 Quality Experiments for varying value of $M$.

When $\mathrm{M}=0$ it represents traditional collaborative filtering system and when $\mathrm{M}=1$ it shows fully maintained pyramid. For $M$ values between zero and one, the quality improvement of LAS increases for higher values of $\mathrm{M}$ due to the increase

in recommendation locality. LAS achieves better quality improvement over LARS because LAS maintains $\alpha$-Cells at lower levels of the pyramid.

\section{Recommendation Quality using MAE}

Fig.shows quality improvement at varying ratio of training and test dataset(variable $\mathrm{x}$ ). at $\mathrm{x}=0.5$ value of MAE is 0.0156 .

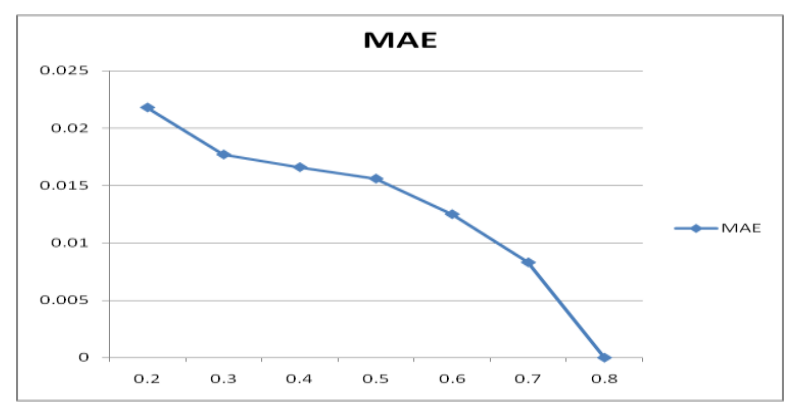

Fig.9 Quality Experiments for MAE.

When value of $x=0.8$ it almost tend to 0.i.e. if the density of dataset increase it shows better performance without loosing the quality.

\section{Recommendation Quality using MAE}

Fig.depicts the storage and aggregate maintenance overhead required for an increasing number of ratings. We again plot LAS-M $=0$ and LAS $M=1$ to indicate the extreme cases for LAS. As shown in fig. the impact of increasing the number of ratings from $10 \mathrm{~K}$ to $500 \mathrm{~K}$ on storage overhead. LAS-M=0 requires the lowest amount of storage since it only maintains a single collaborative filtering model. LAS-M $=1$ requires the highest amount of storage since it requires storage of a collaborative filtering model for all cells (in all levels) of a complete pyramid. The storage requirement of LAS is in between the two extremes since it merges cells to save storage. 


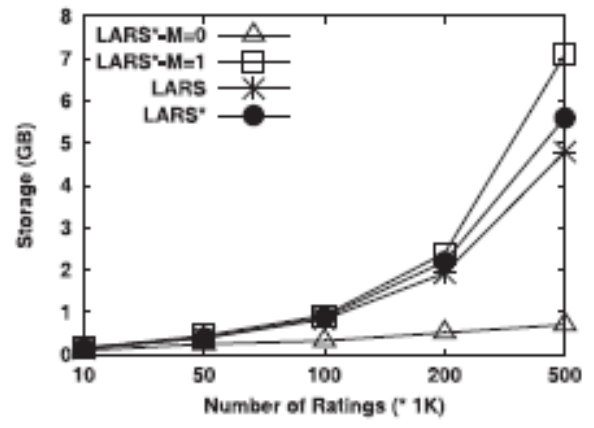

Fig.10[8] Scalability of adaptive pyramid using storage.

\section{CONCLUSION}

LAS overcomes the problem faced by traditional recommender system. It uses User partitioning technique and travel penalty to concern with spatial ratings and spatial item ratings. Algorithm provides efficiency for sclability and locality. As CF model is maintained depending upon the number of $\alpha$-cells in the pyramid it assuares quality of recommendations as well as fast response time as number of user goes on increasing.

\section{REFERENCES}

[1] G. Linden, B. Smith, and J. York, "Amazon.com recommenda-tions: Item-to-item collaborative filtering," IEEE Internet Comput., vol. 7, no. 1, pp. 76-80, Jan./Feb. 2003.

[2] P. Resnick, N. Iacovou, M. Suchak, P. Bergstrom, and J. Riedl, "GroupLens: An open architecture for collaborative filtering of netnews," in Proc. CSWC, Chapel Hill, NC, USA, 1994.

[3] The facebook blog. Facebook Places [Online]. Available: http://tinyurl.com/3aetfs3

[4] G. Adomavicius and A. Tuzhilin, "Toward the next generation of recommender systems: A survey of the state-of-the-art and pos-sible extensions," IEEE Trans. Knowl. Data Eng., vol. 17, no. 6, pp. 734-749, Jun. 2005.

[5] MovieLens [Online]. Available: http://www.movielens.org/

[6] Foursquare [Online]. Available: http://foursquare.com

[7] New York Times - A Peek into Netflix Queues [Online]. Available:

http://www.nytimes.com/interactive/2010/01/10/nyregio n/ 20100110-netflix-map.html

[8] J. J. Levandoski, M. Sarwat, A. Eldawy, and M. F. Mokbel, "LARS: A location-aware recommender system," in Proc. ICDE, Washington, DC, USA, 2012.

[9] B. Sarwar, G. Karypis, J. Konstan, and J. Riedl, "Itembased collab-orative filtering recommendation algorithms," in Proc. Int. Conf. $W W W$, Hong Kong, China, 2001.

[10] J. S. Breese, D. Heckerman, and C. Kadie, "Empirical analysis of predictive algorithms for collaborative filtering," in Proc. Conf. UAI, San Francisco, CA, USA, 1998.
[11] W. G. Aref and H. Samet, "Efficient processing of window queries in the pyramid data structure," in Proc. ACM Symp. PODS, New York, NY, USA, 1990.

[12] R. A. Finkel and J. L. Bentley, "Quad trees: A data structure for retrieval on composite keys," Acta Inf., vol. 4, no. 1, pp. 1-9, 1974.

[13] A. Guttman, "R-trees: A dynamic index structure for spatial searching," in Proc. SIGMOD, New York, NY, USA, 1984.

[14] K. Mouratidis, S. Bakiras, and D. Papadias, "Continuous monitor-ing of spatial queries in wireless broadcast environments," IEEE Trans. Mobile Comput., vol. 8, no. 10, pp. 1297-1311, Oct. 2009.

[15] K. Mouratidis and D. Papadias, "Continuous nearest neighbor queries over sliding windows," IEEE Trans. Knowl. Data Eng., vol. 19, no. 6, pp. 789-803, Jun. 2007.

[16] M. F. Mokbel, X. Xiong, and W. G. Aref, "SINA: Scalable incremental processing of continuous queries in spatiotemporal databases," in Proc. SIGMOD, Paris, France, 2004.

[17] J. L. Herlocker, J. A. Konstan, L. G. Terveen, and J. T. Riedl, "Evaluating collaborative filtering recommender systems," ACM TOIS, vol. 22, no. 1, pp. 5-53, 2004.

[18] M. J. Carey and D. Kossmann, "On saying "Enough Already!" in SQL," in Proc. SIGMOD, New York, NY, USA, 1997.

[19] S. Chaudhuri and L. Gravano, "Evaluating top-k selection queries," in Proc. Int. Conf. VLDB, Edinburgh, U.K., 1999.

[20] R. Fagin, A. Lotem, and M. Naor, "Optimal aggregation algo-rithms for middleware," in Proc. ACM Symp. PODS, New York, NY, USA, 2001.

[21] J. Bao, C.-Y. Chow, M. F. Mokbel, and W.-S. Ku, "Efficient evalu-ation of $\mathrm{k}$-range nearest neighbor queries in road networks," in Proc. Int. Conf. MDM, Kansas City, MO, USA, 2010.

[22] G. R. Hjaltason and H. Samet, "Distance browsing in spatial databases," ACM TODS, vol. 24 , no. 2, pp. 265 318, 1999.

[23] K. Mouratidis, M. L. Yiu, D. Papadias, and N. Mamoulis, "Continuous nearest neighbor monitoring in road networks," in Proc. Int. Conf. VLDB, Seoul, Korea, 2006.

[24] D. Papadias, Y. Tao, K. Mouratidis, and C. K. Hui, "Aggregate nearest neighbor queries in spatial databases," ACM TODS, vol. 30, no. 2, pp. 529-576, 2005.

[25] S. Börzsönyi, D. Kossmann, and K. Stocker, "The skyline opera-tor," in Proc. ICDE, Heidelberg, Germany, 2001.

[26] M. Sharifzadeh and C. Shahabi, "The spatial skyline queries," in Proc. Int. Conf. VLDB, Seoul, Korea, 2006.

[27] N. Bruno, L. Gravano, and A. Marian, "Evaluating top-k queries over web-accessible databases," in Proc. ICDE, San Jose, CA, USA, 2002. 
[28] P. Venetis, H. Gonzalez, C. S. Jensen, and A. Y. Halevy, "Hyper-local, directions-based ranking of places," PVLDB, vol. 4, no. 5, pp. 290-301, 2011.

[29] M.-H. Park, J.-H. Hong, and S.-B. Cho, "Location-based recom-mendation system using Bayesian user's preference model in mobile devices," in Proc. Int. Conf. UIC, Hong Kong, China, 2007.

[30] Netflix News and Info - Local Favorites [Online]. Available: http://tinyurl.com/4qt8ujo

[31] Y. Takeuchi and M. Sugimoto, "An outdoor recommendation system in based on user location history," in Proc. Int. Conf. UIC, Berlin, SIGMOD, Germany, 2006.

[32] V. W. Zheng, Y. Zheng, X. Xie, and Q. Yang, "Collaborative loca-tion and activity recommendations with GPS history data," in Proc. Int. Conf. WWW, New York, NY, USA, 2010.

[33] M. Ye, P. Yin, and W.-C. Lee, "Location recommendation for location-based social networks," in Proc. ACM GIS, New York, NY, USA, 2010. 